Amanda Arantes Perez ${ }^{1}$

DÉBORA BALABRAM ${ }^{2}$

Marcio de Almeida Salles ${ }^{3}$

HeLENICE GobBI ${ }^{4}$

Artigo Original

Palavras-chave

Mama/patologia

Diagnóstico histopatológico,

Variabilidade interobservador

Hiperplasia/diagnóstico

Carcinoma intraductal infiltrante/diagnóstico

Keywords

Breast/pathology

Histophatological diagnosis, Interobserver variability

Hyperplasia/diagnosis

Carcinoma, Intraductal, Noninfiltrating/diagnosis

\section{Consultoria em patologia cirúrgica mamária: variabilidade interobservador no diagnóstico de lesões proliferativas intraductais atípicas}

\author{
Consultation in breast surgical pathology: interobserver diagnostic \\ variability of atypical intraductal proliferative lesions
}

Resumo

OBJETIVO: Avaliar a concordância nos diagnósticos histopatológicos de lesões mamárias proliferativas intraductais entre patologistas gerais e especialistas em patologia mamária. MÉTODOS: Trata-se de estudo observacional e transversal, com análise de 209 lesões encaminhadas ao Laboratório de Patologia Mamária da Faculdade de Medicina da Universidade Federal de Minas Gerais para consultoria, no período de 2007 a 201 1, comparando os diagnósticos originais com os após a revisão. Foram incluídos apenas os casos com solicitação formal de revisão e que apresentavam diagnóstico histopatológico no laudo original ou de revisão de lesões proliferativas, carcinoma ductal in situ puro, carcinoma ductal in situ com microinvasão ou associado a carcinoma invasor. A concordância percentual e o índice kappa foram utilizados para a análise estatística. RESULTADOS: Observamos moderada concordância nos diagnósticos originais de benignidade ou malignidade versus os diagnósticos de revisão (kappa=0,5; concordância percentual=83\%). Após a revisão, o diagnóstico de malignidade foi confirmado em 140/163 casos (86\%) e o diagnóstico de benignidade foi confirmado em 34/46 casos (74\%). Quanto aos diagnósticos específicos, observamos concordância moderada entre o laudo original e de revisão (136/209 casos; kappa=0,5; concordância percentual=65\%). A maior discordância foi observada nos casos de carcinoma ductal in situ com microinvasão (6/6 casos; 100\%). Grande discordância foi observada nos casos de hiperplasia ductal atípica (16/30 casos; $53 \%$ ) e carcinoma ductal in situ (25/75 casos; 33\%). Em relação ao grau histológico do carcinoma ductal in situ, observouse boa concordância entre os laudos originais e de revisão (29/39 casos; kappa=0,6; concordância percentual=74\%). CONCLUSÃO: Nossos dados confirmam que as lesões mamárias proliferativas intraductais, em especial as hiperplasias ductais atípicas, o carcinoma ductal in situ e o carcinoma ductal in situ com microinvasão apresentam relevantes discordâncias nos diagnósticos histopatológicos, que podem induzir o clínico a erros nas decisões terapêuticas.

\section{Abstract}

PURPOSE: To evaluate the agreement about the histopathological diagnosis of intraductal proliferative breast lesions between general pathologists and a specialist in breast pathology. METHODS: This was an observational, cross-sectional study of 209 lesions received in consultation at the Breast Pathology Laboratory of the School of Medicine, Federal University of Minas Gerais, from 2007 to 2011 , comparing the original diagnosis and the review. We included only cases with a formal request for review and cases in which the original diagnosis or reviewer's diagnosis showed proliferative lesions, pure ductal carcinoma in situ, ductal carcinoma in situ associated with microinvasion or associated with invasive carcinoma. The kappa index and percent concordance were used in the statistical analyses. RESULTS: A moderate agreement was observed between the original histopathological diagnosis and the second opinion (kappa $=0.5$; percentual concordance $=83 \%$ ). After the review, the diagnosis of malignancy was confirmed in 140/163 cases (86\%) and the diagnosis of benign lesions was confirmed in 34/46 cases $(74 \%)$. Regarding specific diagnosis, we observed moderate agreement between the original diagnosis and the reviewer's diagnosis (136/209 cases; kappa=0.5; percent concordance=65\%). The highest disagreement was observed in cases of ductal carcinoma in situ with microinvasion (6/6 cases; 100\%). Important discordance was observed in cases of atypical ductal hyperplasia (16/30 cases; 53\%) and ductal carcinoma in situ (25/75 cases; 33\%). Regarding the histological grade of ductal carcinoma in situ, we observed good agreement between the original diagnosis and the review (29/39 cases; kappa=0.6, percent agreement=74\%). CONCLUSION: Our data confirm that intraductal proliferative breast lesions, especially atypical ductal hyperplasia, ductal carcinoma in situ and ductal carcinoma in situ with microinvasion show relevant discrepancies in the histopathological diagnoses, which may induce errors in therapeutic decisions.
Correspondência

Helenice Gobbi

Avenida Prof. Alfredo Balena, 190 - Santa Efigênic

CEP: $30130-100$

Belo Horizonte (MG), Brasi

Recebido:

$25 / 03 / 2013$
Trabalho realizado no laboratório de Patologia Mamária da Faculdade de Medicina, Universidade Federal de Minas Gerais - UFMG Belo Horizonte (MG), Brasil.

IPrograma de Pós-Graduação em Saúde da Mulher, Universidade Federal de Minas Gerais - UFMG - Belo Horizonte (MG), Brasil. 2Programa de Pós-Graduação em Patologia, Universidade Federal de Minas Gerais - UFMG - Belo Horizonte (MG), Brasil.

${ }^{3}$ Hospital Vila da Serra - Belo Horizonte (MG), Brasil.

${ }^{4}$ Departamento de Anatomia Patológica e Medicina Legal da Faculdade de Medicina, Universidade Federal de Minas Gerais - UFMG Belo Horizonte (MG), Brasil.

Conflito de interesse: não há 
Introdução

A frequência de lesões proliferativas intraductais atípicas e do carcinoma ductal in situ da mama (CDIS) apresentou aumento significativo nos últimos 20 anos, principalmente após a introdução de programas de rastreamento utilizando a mamografia ${ }^{1,2}$. Ginecologistas e mastologistas têm-se deparado com estes diagnósticos de forma crescente também em nosso meio. A conduta terapêutica varia de acordo com o tipo de lesão proliferativa e o risco relativo para evolução para carcinoma invasor associado a elas.

Em anatomia patológica, a baixa variação interobservador geralmente funciona como um substituto para a avaliação da acurácia, e os erros são classificados em "maior" ou "menor" conforme a repercussão no tratamento ou no prognóstico da doença. "Erro maior" constitui erro que tenha efeito maior na terapia ou que possa alterar o prognóstico da doença; "erro menor" compreende erro sem efeito maior na terapia ou no prognóstico da doença ${ }^{3}$.

O diagnóstico diferencial entre as lesões mamárias proliferativas intraductais atípicas CDIS e CDIS com microinvasão pode, algumas vezes, ser difícil para os patologistas gerais e o tratamento modifica-se significativamente com base no diagnóstico final do patologista. Tais lesões têm sido objeto de estudos que mostram concordância diagnóstica variável e dificuldade na reprodução dos diagnósticos histopatológicos entre patologistas da comunidade e especialistas em patologia mamária ${ }^{4-9}$.

Estudo anterior de nosso grupo avaliou uma série de casos de lesões mamárias recebidos para revisão no período de 1999 a 2006. Observamos maior dificuldade e maior discordância no diagnóstico do CDIS e das lesões proliferativas intraductais, comparado aos diagnósticos de carcinomas invasores, para os quais há ótima concordância diagnóstica ${ }^{8}$

O objetivo do presente estudo foi avaliar a concordância nos diagnósticos histopatológicos de lesões mamárias proliferativas intraductais entre patologistas gerais e especialistas em patologia mamária, em casos recebidos em consultoria, com ênfase nos casos de hiperplasia ductal atípica (HDA), CDIS e CDIS com microinvasão, em um período de abrangência cinco anos posterior ao primeiro estudo.

\section{Métodos}

Trata-se de estudo observacional do tipo transversal, com análise de casos recebidos em consultoria no Laboratório de Patologia Mamária da Faculdade de Medicina da Universidade Federal de Minas Gerais no período de 2007 a 2011 para obtenção de segunda opinião.

Os critérios de inclusão no estudo foram: casos encaminhados para consultoria em patologia mamária contendo solicitação formal de revisão e cópia do laudo anatomopatológico original; diagnóstico histopatológico de lesões proliferativas intraductais (hiperplasia ductal usual, lesão de células colunares, atipia plana, HDA), CDIS puro, CDIS com microinvasão ou CDIS associado a carcinoma invasor nos laudos anatomopatológicos originais ou de revisão; casos em que a revisão foi realizada por única patologista (HG), especialista em patologia cirúrgica mamária e responsável pelo Serviço de Consultoria em Patologia Mamária há 24 anos. Os critérios de exclusão foram: casos de solicitação informal de revisão anatomopatológica, ou seja, que não apresentavam pedido formal de revisão; ausência de cópia do laudo anatomopatológico original; casos em que a revisão foi realizada por outro patologista do serviço; casos com diagnóstico original de neoplasia lobular in situ (NLIS), tumores fibroepiteliais (fibroadenomas, tumores phyllodes, hamartomas), proliferações epiteliais benignas (adenose esclerosante, adenose apócrina, adenose microglandular, cicatriz radial, adenoma tubular, adenoma da lactação, adenoma apócrino, adenoma ductal), lesões papilares sem atipias ou neoplasia invasora pura (não associada a CDIS).

Foram selecionados inicialmente 388 casos com requisição formal de revisão e cópia dos laudos anatomopatológicos originais, avaliados por única patologista (HG). Destes, foram excluídos 62 casos com diagnóstico original de lesão benigna, incluindo tumores fibroepiteliais, proliferações epiteliais benignas, lesões papilares sem atipias e NLIS. Também foram excluídos 117 casos com diagnóstico original de carcinoma invasor puro (não associado a CDIS). Foram avaliados, portanto, 209 casos que apresentavam lesão proliferativa intraductal, CDIS puro, CDIS com microinvasão ou CDIS associado a carcinoma invasor como diagnóstico histopatológico no laudo original ou de revisão.

Avaliamos a procedência geográfica do material original, o tipo do espécime analisado e o solicitante da segunda opinião, sendo este categorizado como paciente/familiar ou médico assistente segundo a especialidade (Mastologista, Oncologista ou Patologista). Os casos não classificados nas categorias descritas anteriormente foram categorizados como "outros solicitantes".

Aplicando-se estes critérios, a amostra em estudo ficou constituída por pacientes com idades variando de 20 a 86 anos (média de 51,7 anos; desvio padrão=11,4 anos). As solicitações de revisão anatomopatológica procederam, em sua maioria, de Belo Horizonte (170/209 casos; 81,4\%), seguida do interior de Minas Gerais (31/209 casos; 14,8\%) e de outros estados (08/209 casos; 3,8\%). Os espécimes recebidos foram provenientes de setorectomias (174/209 casos; 83,3\%), core biopsy ou biópsias incisionais (27/209 casos; $12,9 \%)$ e mastectomias (08/209 casos; $3,8 \%$ ). Os mastologistas foram responsáveis por $84,7 \%$ das requisições (177/209 casos), seguidos dos patologistas 
(15/209 casos; 7,1\%) e oncologistas (14/209 casos; 6,7\%). Apenas uma revisão foi solicitada pelo paciente ou familiar $(0,5 \%)$ e duas requisições foram encaminhadas por outros solicitantes (1\%).

Os diagnósticos originais e de revisão foram inicialmente classificados como benignos ou malignos. Segundo a classificação da Organização Mundial de Saúde ${ }^{10}$, as lesões foram agrupadas conforme o diagnóstico histopatológico específico: lesões benignas, incluindo tumores fibroepiteliais, proliferações epiteliais benignas e lesões papilares sem atipias; lesões proliferativas intraductais, incluindo hiperplasia ductal usual, lesão de células colunares e atipia plana; HDA; NLIS; CDIS; CDIS com microinvasão e carcinoma invasor. O grau histológico também foi avaliado nos casos de CDIS ${ }^{10}$.

Os critérios de Page e Anderson (1987) ${ }^{11}$ foram utilizados pela consultora (HG) para classificação da HDA e do CDIS. Hiperplasia ductal atípica foi caracterizada pela proliferação de células monofórmicas, com distribuição regular, formando lumens secundários regulares, arredondados e uniformes. As lesões são pequenas e as células envolvem parcialmente dois ductos ou "espaços", ou medem menos que dois milímetros. O CDIS é definido como proliferação epitelial de células atípicas, que envolve completamente dois "espaços" ou ductos, ou mede em conjunto mais de dois milímetros ${ }^{11}$. Para o diagnóstico de CDIS com microinvasão foram utilizados os critérios de Ellis et al. (1998) ${ }^{12}$. O diagnóstico de microinvasão compreende CDIS predominantemente e infiltração de células neoplásicas além da membrana basal no tecido conjuntivo não especializado ou extralobular até um milímetro na maior dimensão. Para o diagnóstico da NLIS foram utilizados os critérios propostos pela $\mathrm{OMS}^{10}$. O grau histológico do CDIS foi determinado utilizando os critérios de Scott et al. ${ }^{13}$, considerando o grau de atipia nuclear, a presença e a extensão da necrose.

A concordância percentual e o índice kappa foram utilizados para a análise da variação interobservador (diagnóstico original versus diagnóstico de revisão), sendo considerados os seguintes valores: $<0,2$ (ruim); 0,2-0,39 (fraca); 0,4-0,59 (moderada); 0,6-0,79 (boa); 0,8-1,00 (excelente) ${ }^{9}$.

Este trabalho foi aprovado pelo Comitê de Ética em Pesquisa da Universidade Federal de Minas Gerais (parecer ETIC 655/08).

\section{Resultados}

Dos 209 casos avaliados, 163 lesões foram classificadas como malignas ( $78 \%$ da amostra) e 46 lesões (22\% da amostra) foram consideradas benignas no diagnóstico original. Após a revisão, o diagnóstico de malignidade foi confirmado em 140/163 casos (86\%) e o diagnóstico de benignidade, em 34/46 casos (74\%). Vinte e três lesões caracterizadas inicialmente como malignas (23/163 casos; $14 \%$ ) foram consideradas benignas pela consultora e 12/46 lesões (26\%) originalmente classificadas como benignas foram consideradas malignas após a revisão. Observou-se moderada concordância nos diagnósticos originais de benignidade ou malignidade versus os diagnósticos de revisão (kappa $=0,5$; concordância percentual global $=83 \%$; Tabela 1 ).

Quanto aos diagnósticos específicos, também observamos concordância moderada entre o laudo original e de revisão (136/209 casos; kappa=0,5; concordância percentual $=65 \%$; Tabela 2 ). Houve discordância diagnóstica em 73/209 casos (35\%). Com o diagnóstico de revisão, possível mudança terapêutica ocorreria em $27 \%$ dos casos (56/209 casos). Doze pacientes não teriam sido submetidas a tratamento oncológico (12/209 casos com diagnóstico original de lesão benigna, classificadas como lesão maligna após a revisão; 5,7\%). Ao contrário, 23/209 casos (11\%) classificados originalmente como malignos foram considerados benignos após a revisão. Treze pacientes $(6,2 \%)$ teriam sido submetidas à abordagem axilar desnecessária e cinco pacientes $(2,4 \%)$ não teriam sido submetidas à abordagem axilar após a revisão histopatológica.

Discordância importante foi observada nos diagnósticos de HDA (16/30 casos; 53\%). Após a revisão, quatro lesões classificadas originalmente como HDA foram consideradas CDIS e uma lesão foi classificada como carcinoma invasor. Também observamos grande discordância nos diagnósticos de CDIS (25/75 casos; 33\%) e CDIS com microinvasão (6/6 casos; $100 \%)$. Dos 75 casos com diagnóstico original de CDIS, 20 casos foram classificados como lesão benigna após a revisão (27\%), duas lesões foram avaliadas como CDIS com microinvasão e três lesões foram reclassificadas como carcinoma invasor. Dos seis casos classificados originalmente como CDIS com microinvasão, três casos foram considerados CDIS sem microinvasão e três casos foram reclassificados como carcinoma invasor (Figura 1).

Em relação ao grau histológico do CDIS, observou-se boa concordância entre os laudos originais e de revisão (29/39 casos; índice de kappa =0,6; concordância percentual $=74 \%$ ). Em 11 casos o grau histológico do CDIS não foi descrito no laudo original, impossibilitando a comparação com o laudo de revisão. O alto grau histológico no

Tabela 1. Distribuição dos casos de acordo com o diagnóstico original e após a revisão, segundo as categorias: benigno ou maligno

\begin{tabular}{lccc}
\hline Diagnóstico & \multicolumn{3}{c}{ Diagnóstico após revisão } \\
\cline { 2 - 4 } original & Benigno & Maligno & Tołal \\
\hline Benigno & 34 & 12 & 46 \\
Maligno & 23 & 140 & 163 \\
Tołal & 57 & 152 & 209 \\
\hline
\end{tabular}

Índice de kappa: 0,55; concordância percentual global: 83\%. 
diagnóstico original foi confirmado em todos os casos após a revisão (14/39 casos). Dos 15 casos que apresentavam baixo grau histológico no diagnóstico original, nenhum foi reclassificado como alto grau após a revisão. Dois casos foram considerados de grau intermediário após a revisão.

\section{Discussão}

Estudos anteriores evidenciaram que as lesões proliferativas intraductais, em especial as hiperplasias ductais atípicas, o CDIS e o CDIS com microinvasão, apresentam dificuldades de diagnóstico histopatológico e concordância inter e intraobservador variável na literatura ${ }^{4-9}$. A conduta ou decisão terapêutica proposta pelo médico assistente depende, intrinsecamente, do diagnóstico emitido pelo patologista no laudo histopatológico. Portanto, um diagnóstico errôneo de uma lesão proliferativa intraductal pode acarretar em erro maior ou menor no processo de cuidado do paciente.

No presente estudo, observamos concordância moderada entre os diagnósticos de malignidade e benignidade nos

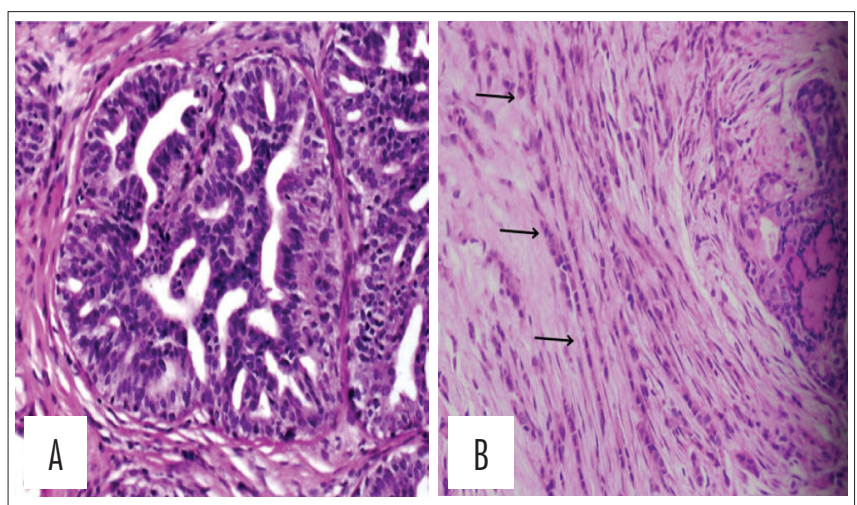

Figura 1. (A) Hiperplasia ductal atípica originalmente diagnosticada como carcinoma ductal in situ. Os dois ductos são incompletamente preenchidos pelas células atípicas. (B) Carcinoma ductal in situ com microinvasão no diagnóstico original. A dimensão da lesão invasiva (setas), maior que $1 \mathrm{~mm}$, favoreceu o diagnóstico de carcinoma ductal invasor na revisão. laudos originais e de revisão. Divergências diagnósticas entre lesões benignas versus malignas configuram erros diagnósticos maiores, com repercussão no tratamento e/ou prognóstico dos pacientes. Lesões malignas classificadas erroneamente como benignas impossibilitam o tratamento oncológico adequado, acarretando prejuízos graves ao paciente. Por outro lado, lesões benignas classificadas erroneamente como malignas resultam em tratamentos cirúrgicos e oncológicos desnecessários, com aumento da morbidade. Estudo anterior de nosso grupo avaliando o diagnóstico de lesões mamárias em geral evidenciou boa concordância entre os diagnósticos originais e de revisão quanto à classificação benigno versus maligno ${ }^{8}$. O maior índice de concordância observado no estudo anterior pode, provavelmente, ser explicado pela inclusão de casos de lesões benignas não proliferativas e neoplasias invasivas puras. No estudo atual focalizamos nossa avaliação nas lesões que são consideradas na literatura de maior dificuldade no diagnóstico diferencial e referidas por alguns autores como lesões borderline $e^{4,5,9}$.

Com relação à comparação dos diagnósticos específicos (originais versus de revisão), também observamos, no presente estudo, moderada concordância, semelhante à observada no estudo anterior de nosso grupo ${ }^{8}$. Após a revisão, possível mudança terapêutica ocorreria em $27 \%$ dos casos, com prejuízos relevantes, por subtratamento ou tratamentos desnecessários e excessivos. Sabe-se que o treinamento e uso de critérios padronizados aprimoram o diagnóstico das lesões mamárias borderline e melhoram a concordância interobservador ${ }^{5,7}$.

Nossos resultados mostraram maior discordância no diagnóstico de CDIS com microinvasão. As alterações responsáveis por erros na interpretação diagnóstica foram: presença de denso infiltrado inflamatório, neoformação vascular e extensão da neoplasia para unidades lobulares (referido como cancerização de lóbulos). Em estudo anterior, também observamos maior discordância diagnóstica nos casos de CDIS com microinvasão ${ }^{8}$.

Tabela 2. Distribuição dos casos de acordo com o diagnóstico original e após a revisão, estratificando os diagnósticos específicos

\begin{tabular}{|c|c|c|c|c|c|c|c|c|}
\hline \multirow{2}{*}{ Diagnóstico original } & \multicolumn{8}{|c|}{ Diagnóstico após revisão } \\
\hline & LB & LP & HDA & NLIS & CDIS & MIC & $\mathrm{Cl}$ & Total \\
\hline LB & 0 & 0 & 2 & 0 & 2 & 0 & 0 & 4 \\
\hline LP & 1 & 3 & 3 & 0 & 3 & 0 & 0 & 10 \\
\hline HDA & 1 & 7 & 14 & 3 & 4 & 0 & 1 & 30 \\
\hline NLIS & 0 & 0 & 0 & 1 & 2 & 0 & 0 & 3 \\
\hline CDIS & 0 & 9 & 9 & 2 & 50 & 2 & 3 & 75 \\
\hline Cl & 0 & 2 & 1 & 0 & 7 & 3 & 68 & 81 \\
\hline Total & 2 & 21 & 29 & 6 & 71 & 5 & 75 & 209 \\
\hline
\end{tabular}

LB: lesão benigna (tumores fibroepiteliais, proliferações epiteliais benignas, lesões papilares sem atipias); LP: lesão proliferativa intraductal (hiperplasia ductal usual, lesão de células colunares, atipia plana); HDA: hiperplasia ductal atípica; NLIS: neoplasia lobular in situ; CDIS: carcinoma ductal in situ; MIC: carcinoma ductal in situ com microinvasão; $\mathrm{Cl}$ : carcinoma invasor. Números em negrito indicam discordâncias diagnósticas (73/209 casos; 35\%). Números sublinhados indicam possíveis mudanças terapêuticas (56/209 casos; 27\%). Índice de kappa: 0,5; concordância percentual global: 65\%. 
No presente estudo, discordância relevante foi observada no diagnóstico de HDA, com mudança substancial no tratamento em cinco casos (17\%). Concordância moderada entre os diagnósticos original e de revisão nos casos de HDA também foi observada em nosso estudo anterior $(\mathrm{kappa}=0,5)^{8}$. Apesar deste tópico ser abordado em várias publicações, o diagnóstico diferencial entre HDA e CDIS de baixo grau e, algumas vezes, HDA versus hiperplasia ductal usual permanece um desafio para os patologistas. A HDA é definida como uma entidade que apresenta algumas, porém não todas as características do CDIS. As características morfológicas e as medidas quantitativas para definição do diagnóstico de HDA são subjetivas e ainda não há consenso sobre os critérios que deveriam ser utilizados para o diagnóstico ${ }^{14}$. Jain et al..$^{9}$ observaram fraca concordância interobservador no diagnóstico de 81 lesões proliferativas avaliadas por nove patologistas diferentes (kappa $=0,3)$, com melhora significativa desta concordância após a realização de estudo imuno-histoquímico (kappa $=0,5 ; \mathrm{p}=0,015)$.

Neste estudo, após a revisão, observamos discordância diagnóstica em $33 \%$ dos casos de CDIS, acarretando, em alguns casos, mudanças significativas no tratamento cirúrgico e adjuvante e, em outros, contraindicando tratamentos cirúrgicos agressivos, radioterapia e/ou hormonioterapia desnecessários. Em estudo anterior, observamos concordância interobservador desprezível ou mínima entre especialista internacional e cinco patologistas gerais da comunidade (kappa $=0,09$ a 0,39 ) no diagnóstico de $\mathrm{CDIS}^{6}$. A diferença de resultados entre o estudo atual e o anterior pode ser explicada pelos critérios de seleção. No presente estudo, selecionamos casos consecutivos encaminhados para consultoria em patologia cirúrgica mamária, com diagnóstico histopatológico de lesão proliferativa intraductal, com ou sem atipia, CDIS puro, CDIS com microinvasão ou associado a carcinoma invasor no laudo original ou de revisão. No estudo anterior, foram selecionados apenas casos que apresentavam divergências no diagnóstico de CDIS entre patologistas da comunidade e a consultora (HG). Os casos incluídos no estudo prévio foram reavaliados por especialista internacional (David L. Page, da Vanderbilt University, EUA), que foi considerado o diagnóstico de referência ou padrão-ouro nas comparações. Além disto, todos os casos incluídos eram de difícil diagnóstico, o que justificava a maior variação interobservador. Ainda na mesma linha de pesquisa, em trabalho posterior, avaliamos casos consecutivos encaminhados para consultoria em patologia mamária e observamos percentual de discordância no diagnóstico de CDIS semelhante ao descrito no presente estudo (percentual de discordância $=36 \%)^{8}$. Se considerarmos que o estudo atual analisou casos diagnosticados em um período de cinco anos após nosso primeiro estudo, podemos afirmar que a dificuldade diagnóstica das lesões avaliadas persiste em nosso meio.

O grau histológico é considerado preditor do comportamento biológico do CDIS, especialmente por sua associação com o risco de desenvolvimento de carcinoma invasor ${ }^{15}$. Poucos estudos avaliaram a concordância interobservador e a reprodutibilidade dos critérios de classificação do grau histológico do CDIS. No presente estudo, observamos boa concordância na avaliação do grau histológico do CDIS. O alto grau histológico no diagnóstico original foi confirmado em todos os casos após a revisão. Em estudo prévio, observamos concordância moderada ( $k a p p a=0,5 ;$ concordância percentual $=68,8 \%$ ) na avaliação do grau nuclear do CDIS, não sendo avaliado o grau histológico das lesões ${ }^{8}$. Douglas-Jones et al. ${ }^{16}$ relataram moderada concordância interobservador entre patologistas não especialistas em patologia mamária em relação à avaliação do grau histológico do CDIS, com melhor concordância para a avaliação de necrose em relação ao grau nuclear.

$\mathrm{Na}$ classificação mais recente de tumores mamários da OMS (2012), as lesões mamárias são classificadas como tumores epiteliais, incluindo o carcinoma microinvasor e os carcinomas invasores; lesões precursoras (CDIS, NLIS); lesões proliferativas intraductais (hiperplasia ductal usual, lesão de células colunares, atipia plana, HDA); lesões papilares; proliferações epiteliais benignas (adenose esclerosante, adenose apócrina, adenose microglandular, adenomas, cicatriz radial) e tumores fibroepiteliais (fibroadenomas, tumores phyllodes e hamartomas $)^{10}$. Para Masood e Rosa ${ }^{14}$, os critérios propostos por Dupont e Page $(1985)^{17}$, considerando o risco para o desenvolvimento de câncer de mama, representam a maneira mais adequada de classificar as lesões borderline da mama, considerando-se lesões mamárias não proliferativas (cistos, hiperplasias leves), lesões proliferativas sem atipias (fibroadenoma complexo, hiperplasia moderada florida, adenose esclerosante, papiloma intraductal) e lesões proliferativas com atipias (HDA, HLA) ${ }^{14}$. No presente estudo, optamos por agrupar as lesões mamárias conforme a classificação mais recente proposta pela OMS. Para facilitar a análise, as lesões papilares sem atipias, as proliferações epiteliais benignas e as lesões proliferativas intraductais, excluindo a HDA, foram agrupadas sob o termo "lesões benignas". Com o objetivo de enfatizar e discriminar a variabilidade interobservador especificamente nos diagnósticos de HDA, NLIS e CDIS, tais lesões foram analisadas separadamente. O diagnóstico de HDA em biópsia de mama significa aumento do risco relativo de desenvolvimento de câncer de mama em aproximadamente 4 a 5 vezes, diferente dos diagnósticos de hiperplasia ductal usual, lesão de células colunares ou 
atipia plana, considerados de risco leve (1,2 a 2 vezes). Dentro das lesões precursoras, a NLIS é considerada um fator de risco e um precursor não obrigatório para o câncer de mama; a conduta pode variar desde apenas acompanhamento clínico até a realização de mastectomia profilática e uso de hormonioterapia ${ }^{14}$. O tratamento do CDIS é definido considerando o potencial de evolução e recorrência da lesão; pode variar de uma ressecção segmentar isolada ou associada à radioterapia, até a realização de mastectomia ${ }^{18}$.

No presente estudo, apenas os casos encaminhados para consultoria em patologia mamária contendo solicitação formal de revisão e cópia do laudo anatomopatológico original foram incluídos, tendo em vista que a ausência da cópia do laudo original impossibilitaria a comparação dos diagnósticos original e final. Casos de solicitação informal de revisão, trazidos por colegas patologistas foram recebidos com maior frequência no Laboratório de Patologia Mamária, mas não foram incluídos no estudo. Optamos por não incluir os casos com diagnóstico original de NLIS, tumores fibroepiteliais, lesões papilares sem atipias ou neoplasia invasora pura, com o objetivo de enfatizar especificamente o diagnóstico histopatológico de lesões proliferativas intraductais, principalmente os casos de HDA, CDIS e CDIS com microinvasão.

Patologistas foram responsáveis por apenas $7,1 \%$ das solicitações formais de revisão. Recorrer formalmente a interconsultas com especialistas não é prática comum entre os patologistas em nosso meio. Muitos preferem realizar consultas informais, solicitadas como "gentileza" aos colegas especialistas, sem registrar no laudo que houve discussão do caso com outros colegas ou que seria importante a obtenção de segunda opinião ${ }^{19}$. Ao contrário do Brasil, nos Estados Unidos a solicitação de segunda opinião em patologia é prática comum e muitos pedidos de segunda opinião são solicitados pelos próprios patologistas. Através de interconsultas, os médicos americanos procuram se resguardar de possíveis processos contra "má prática" ou erro médico, cuja consequência legal pode afetar todos os profissionais envolvidos no diagnóstico e tratamento do paciente 20-22 $^{\text {. }}$

Em conclusão, nossos dados confirmam que as lesões mamárias proliferativas intraductais, em especial as hiperplasias ductais atípicas, o CDIS e o CDIS com microinvasão, apresentam relevantes discordâncias nos diagnósticos histopatológicos, que podem induzir o clínico a erros maiores na conduta terapêutica. Com o uso crescente da mamografia de rastreamento e maior detecção das lesões proliferativas intraductais, torna-se necessário melhor treinamento dos patologistas da comunidade no reconhecimento dessas lesões, resultando, assim, em melhor acurácia diagnóstica. Baseado em nossos resultados, consideramos que a solicitação formal de segunda opinião em lesões mamárias precursoras e borderline deva ser estimulada em nosso meio, principalmente entre os patologistas, com o objetivo de diminuir erros diagnósticos, assegurar a conduta terapêutica adequada e garantir a segurança do paciente.

\section{Agradecimentos}

Este estudo teve suporte de recursos da Fundação de Amparo a Pesquisa de Minas Gerais (FAPEMIG) e Conselho Nacional de Desenvolvimento Científico e Tecnológico (CNPq).

\section{Referências}

1. Burstein HJ, Polyak K, Wong JS, Lester SC, Kaelin CM. Ductal carcinoma in situ of the breast. N Engl J Med. 2004;350(14):1430-41.

2. O'Sullivan M, Morrow M. Ductal carcinoma in situ: current management. Surg Clin North Am. 2007;87(2):333-51.

3. Sirota RL. Defining error in anatomic pathology. Arch Pathol Lab Med. 2006;130(5):604-6.

4. Rosai J. Borderline epithelial lesions of the breast. Am J Surg Pathol. 1991;15(3):209-21.

5. Schnitt SJ, Connolly JL, Tavassoli FA, Fechner RE, Kempson RL, Gelman R, et al. Interobserver reproducibility in the diagnosis of ductal proliferative breast lesions using standardized criteria. Am J Surg Pathol. 1992;16(12):1133-43.

6. Salles MA, Matias MARF, Resende LMP, Gobbi H. [Interobserver variation of the histopathologic diagnosis of ductal carcinoma in situ of the breast]. Rev Bras Ginecol Obstet. 2005;27(1):1-6.
7. Salles MA, Gouvêa AP, Savi D, Figueiredo MA, Tavares Neto $R$, Paula RA, et al. [Training and standardized criteria improve the diagnosis of premalignant breast lesions]. Rev Bras Ginecol Obstet. 2008;30(1 1):550-5. Portuguese.

8. Salles Mde A, Sanches FS, Perez AA, Gobbi H. [Importance of a second opinion in breast surgical pathology and therapeutic implications]. Rev Bras Ginecol Obstet. 2008;30(12):602-8. Portuguese.

9. Jain RK, Mehta R, Dimitrov R, Larsson LG, Musto PM, Hodges KB, et al. Atypical ductal hyperplasia: interobserver and intraobserver variability. Mod Pathol. $2011 ; 24(7): 917-23$.

10. Lakhani SR, Ellis IO, Schnitt SJ, Tan PH, van de Vijver M. WHO classification of tumours of the breast. 4th ed. Lyon: IARC; 2012.

11. Page DL, Anderson TJ. Diagnostic histopathology of the breast. Edinburgh: Churchill Livingstone; 1987. 
12. Ellis IO, Elston CW, Poller DN. Ductal carcinoma in situ. In: Elston $\mathrm{CW}$, Ellis $1 \mathrm{O}$, editors. The breast. Edinburgh: Churchill Livingstone; 1998. p. 249-82.

13. Scott MA, Lagios MD, Axelsson K, Rogers LW, Anderson TJ, Page DL. Ductal carcinoma in situ of the breast: reproducibility of histological subtype analysis. Hum Pathol. 1997;28(8):967-73.

14. Masood S, Rosa M. Borderline breast lesions: diagnostic challenges and clinical implications. Adv Anat Pathol. $2011 ; 18(3): 190-8$.

15. Goldhirsch A, Ingle JN, Gelber RD, Coates AS, Thürlimann B, Senn HJ. Thresholds for therapies: highlights of the St. Gallen International Expert Consensus on the primary therapy of early breast cancer 2009. Ann Oncol. 2009;20(8):1319-29.

16. Douglas-Jones AG, Morgan JM, Appleton MA, Attanoos RL, Caslin A, Champ CS, et al. Consistency in the observation of features used to classify duct carcinoma in situ (DCIS) of the breast. J Clin Pathol. 2000;53(8):596-602.
17. Dupont WD, Page DL. Risk factors for breast cancer in women with proliferative breast disease. N Engl J Med. 1985;312(3): 146-51.

18. Schuh F, Biazús JV, Resetkova E, Benfica CZ, Edelweiss MIA. Reproducibility of tree classification systems of ductal carcinoma in situ of the breast using a web-based survey. Pathol Res Pract. 2010;206(10):705-11.

19. Cooper K. Errors end error rates in surgical pathology: an Association of Directors of Anatomic and Surgical Pathology survey. Arch Pathol Med Lab. 2006; 130(5):607-9.

20. Association of Directors of Anatomic and Surgical Pathology. Consultations in surgical pathology. Am J Surg Pathol. 1993;17(7):743-5.

21. Gupta D, Layfield LJ. Prevalence of inter-institutional anatomic pathology slide review: a survey of current practice. Am J Surg Pathol. 2000;24(2):280-4.

22. Tomaszewski JE, Bear HD, Connally JA, Epstein Jl, Feldman M, Foucar $\mathrm{K}$, et al. Consensus conference on second opinions in diagnostic anatomic pathology. Who, What, and When. Am J Clin Pathol. 2000; 1 14(3):329-35. 